\title{
Un estudio exploratorio sobre el nivel de conocimiento sobre el tamaño del efecto y meta-análisis en psicólogos profesionales españoles
}

\author{
Laura Badenes-Ribera, Dolores Frías-Navarro y Amparo Bonilla-Campos \\ Universitat de València (España)
}

El enfoque de la Práctica Basada en la Evidencia se basa en la utilización de la investigación científica en la toma de decisiones profesionales en un esfuerzo por producir los mejores servicios posibles en la práctica clínica. El nivel de conocimiento sobre los estadísticos del tamaño del efecto y estudios de meta-análisis puede afectar a las decisiones de los profesionales y poner en riesgo la calidad de las intervenciones psicológicas. Este trabajo recoge los resultados de una encuesta on line sobre el grado de conocimiento de estos tópicos entre psicólogos profesionales españoles. La muestra estuvo formada por 77 participantes ( $68.8 \%$ mujeres y $31.2 \%$ hombres), con una edad media de 41.44 años $(D T=9.42)$, y un rango de edad de 25-64 años. Los resultados sugieren que la mayoría de los psicólogos encuestados no tenían un conocimiento adecuado sobre los índices de tamaño del efecto, los estudios de meta-análisis, y los listados de comprobación de la calidad metodológica de los estudios. Por lo tanto, es necesario mejorar la educación estadística de los psicólogos, la cual podría contribuir también en la mejora de la práctica profesional.

Palabras clave: Práctica Basada en la Evidencia, tamaño del efecto, meta-análisis, psicólogos profesionales, educación estadística.

Effect size and meta-analysis in Spanish professional psychologists The Evidence-Based Practice approach is based on the use of scientific research in decision-making in an effort to produce the best possible services in clinical practice. The knowledge level about effect size statistics and meta-analysis studies may affect the decision soft professionals and jeopardize the quality of psychological interventions. This paper presents the result so fan on-line survey on the degree of knowledge of these topics among Spanish practitioner psychologists. The sample consisted of 77 participants (68.8\% women and $31.2 \%$ men), with a mean age of 41.44 years $(S D=9.42)$, with age range of $25-64$ years. The results suggest that most of the psychologists survey eddid not have adequate knowledge about effect size index, meta-analysis studies, or methodological quality checklists. It is therefore necessary to improve the statistical education of practitioner psychologists, which might also contribute to their provement of professional practice.

Keywords: Evidence-Based Practice, effect size, meta-analysis, practitioner psychologists, statistical education.

Correspondencia: Laura Badenes-Ribera. Avda. Blasco Ibáñez, 21. C.P.: 46010. Valencia (España). E-mail: laura.badenes@uv.es 
La Práctica Basada en la Evidencia (PBE) se define como "la integración de la mejor evidencia disponible con la experiencia clínica en el contexto de las características, cultura y preferencias del paciente" (American Psychological Association (APA), Presidencial Grupo de Trabajo sobre la Práctica Basada en la Evidencia, 2006, p. 273). Por definición, la PBE se basa en la utilización de la investigación científica en la toma de decisión es en un esfuerzo por producirlos mejores servicios posibles en la práctica clínica (APA, 2005; Babione, 2010; Daset y Cracco, 2013; Sánchez-Meca, Boruch, Petrosino, y Rosa-Alcázar, 2002). Por lo tanto, el enfoque de la PBE requiere de los profesionales nuevas habilidades como la capacidad para evaluar y jerarquizar la calidad de las investigaciones psicológicas (Beyth-Maron, Fidler, y Cumming, 2008; Frías-Navarro y Pascual-Llobell, 2003).

Dentro de ese proceso de valoración crítica de la evidencia científica es crucial conocer y comprender las medidas del tamaño de efecto como herramienta para interpretar los datos y los estudios de meta-análisis. Las estimaciones del tamaño del efecto proporcionan mayor información para los juicios sobre la importancia clínica y práctica delos resultados de las investigaciones que el valor de $p$ aportado por el procedimiento de la prueba de contraste de la hipótesis nula (Ferguson, 2009). Además, la sexta edición del Manual de la Asociación Americana de Psicología (APA, 2010) refuerza el uso de las estimaciones del tamaño del efecto junto con su intervalo de confianza en la práctica de la investigación para que el lector pueda apreciar la magnitud y precisión de los hallazgos.

Existen docenas de medidas de tamaño de efecto disponibles en la literatura (Kline, 2013). Sin embargo, los psicólogos están más familiarizados con los estadísticos del tamaño de efecto relacionados con las pruebas estadísticas del análisis de la varianza (ANOVA) y del análisis de regresión lineal, como $\eta^{2}$ y $R^{2}$ (Spring, 2007). De hecho, los estadísticos del tamaño de efecto más frecuentemente reportados en las revistas psicológicas son $R^{2}, d$ de Cohen y $\eta^{2}$ (e.g., Badenes-Ribera, Frías-Navarro, Monterde-i-Bort, y Pascual-Soler, 2013; Peng y Chen, 2014). Sin embargo, estos índices del tamaño del efecto han sido criticados por su sesgo (es decir, tienden a estar positivamente sesgados en muestras pequeñas), su falta de robustez a los valores anómalos y, por su inestabilidad bajo las violaciones de los supuestos estadísticos (Grissom y Kim, 2012; Kline, 2013; Wang y Thompson, 2007). Por el contrario, en las ciencias biomédicas (e.g., medicina clínica, epidemiología), por lo general, los hallazgos son expresados como odds ratio, Riesgo Relativo o Número Necesario para Tratar (NNT) (Spring, 2007).

Por último, las revisiones sistemáticas y los estudios meta-analíticos han adquirido considerable relevancia y prevalencia en las revistas más prestigiosas, ya que sintetizan las evidencias disponibles para responder a una pregunta clínica a partir de los resultados de estudios primarios (Bauer, 2007; Borenstein, Hedges, Higgins, y Rothstein, 
2009; Sánchez-Meca y Marín-Martínez, 2010; Walker y London, 2007). Las revisiones sistemáticas y los estudios meta-analíticos ofrecen varias ventajas sobre las revisiones narrativas: las revisiones sistemáticas y los meta-análisis implican un proceso de investigación científicamente basado que depende del rigor y la transparencia de cada una de las decisiones tomadas durante su elaboración (Spring, 2007; Walker y London, 2007). Además, los estudios meta-analíticos pueden proporcionar una respuesta sobre la naturaleza de un efecto cuando hay resultados contradictorios (Bauer, 2007; Borenstein et al., 2009; Botella y Sánchez-Meca, 2015). Y, finalmente, los estudios de meta-análisis facilitan estimaciones más precisas del tamaño del efecto y permiten evaluar la estabilidad de los efectos (Cumming, Fidler, Kalinowski, y Lai, 2012).

Sin embargo, los estudios de meta-análisis no están libres de sesgo (Botella y Sánchez-Meca, 2015). Por ejemplo, Ferguson y Branninck (2011) analizaron 91 estudios meta-analíticos publicados en las revistas American Psychological Association y Association for Psychological Science y encontraron que, de los 91 estudios analizados, $26(41 \%)$ reportaron evidencia de sesgo de publicación. Más recientemente, Kicinski (2013) encontró evidencia del sesgo de publicación en una proporción sustancial de grandes meta-análisis publicados entre 2008 y 2012 en cuatro revistas médicas importantes (British Medical Journal, Journalofthe American Medical Association, Lancet y PlosOne Medicine). Finalmente, Kicinski, Springate, y Kontopantelis (2015) detectaron la presencia de sesgo de publicación en estudios de meta-análisis de la Biblioteca Cochrane que comparaban el tratamiento con placebo o ningún tratamiento.

El sesgo de publicación es una de las mayores amenazas a la validez de los estudios de meta-análisis (Rothstein, Sutton, y Borenstein, 2005). El sesgo de publicación se refiere a la publicación selectiva de estudios que informan resultados estadísticamente significativos, a expensas de estudios con resultados nulos (no estadísticamente significativos). Dado que la mayoría de los estudios de meta-análisis se llevan a cabo con estudios publicados (Clarke y Clarke, 2000), y estos por lo general reportan resultados estadísticamente significativos, el tamaño del efecto medio reportado en un estudio de meta-análisis podría ser sobreestimado, lo que exageraría la importancia de los fenómenos analizados (Borensteinet al., 2009; Cumming, 2012; Ioannidis, 2011). Por lo tanto, el sesgo de publicación puede distorsionar el conocimiento científico sobre temas relacionados con la salud y muchos otros temas de interés científico (Rothstein et al., 2005). Así pues, los lectores de los estudios meta-analíticos (como los psicólogos profesionales) deberían conocer métodos para detectar este sesgo (Bauer, 2007; Spring, 2007). Por ejemplo, el funnelplot (diagrama de embudo) es una representación gráfica que se utiliza frecuentemente como un método de detección de sesgo de publicación en las Ciencias de la Salud (Sterne, Gavaghan, y Egger, 2005). Además, existen listas de verificación para evaluar la calidad de los estudios meta-analíticos (e.g., AMSTAR o PRISMA-NMA). 
Un estudio reciente sobre el nivel de conocimiento sobre las medidas del tamaño de efecto y de los estudios de meta-análisis realizado en una muestra de psicólogos académicos españoles (Badenes-Ribera, Frías-Navarro, Pascual-Soler, y Monterde-i-Bort, 2016) encontró un pobre conocimiento de estos términos entre los psicólogos académicos españoles lo cual podría conducir a una interpretación errónea de los resultados.

Sin embargo, no se sabe nada sobre el nivel de conocimiento metodológico entre los psicólogos profesionales españoles. Los psicólogos necesitan conocer estos conceptos básicos para poder valorar críticamente la relevancia y calidad de la evidencia disponible, con el fin de implementar una mejor práctica. Como señalan Faulkner, Fidler, y Cumming (2008) el valor de la evidencia depende de la calidad de los análisis estadísticos y de su interpretación. Por lo tanto, la interpretación de los hallazgos es un filtro de calidad que no puede ser sometido a creencias erróneas o malas interpretaciones del procedimiento estadístico. En consecuencia, es necesario realizar investigaciones sobre el nivel de conocimiento metodológico que los psicólogos profesionales tienen para desarrollar programas de formación que faciliten o mejoren la implementación del enfoque PBE.

En este sentido, este trabajo analiza el conocimiento metodológico de los psicólogos profesionales de España, a través de un estudio exploratorio. Se espera, en base a estudios previos (e.g., Badenes-Ribera, 2016; Badenes-Ribera et al., 2016), que los profesionales de la psicología tengan un pobre conocimiento acerca de estos tópicos (tamaño del efecto, intervalos de confianza, meta-análisis y listas de comprobación de la calidad metodológica de los estudios). Para ello, realizamos una encuesta on line en la que se invitó a los psicólogos españoles a participar de forma voluntaria y anónima.

\section{MÉTODO}

\section{Participantes}

Se utilizó un muestreo no probabilístico. La muestra inicial estuvo formada por 113 psicólogos españoles. El 68.1\% de los participantes tenía como rol principal el ejercicio profesional de la psicología, el $28.3 \%$ el rol principal de psicólogo académico, el $0.9 \%$ de investigador y el $2.7 \%$ otro rol. Puesto que el objetivo del estudio era analizar las barreras hacia una Práctica profesional Basada en la Evidencia, se eliminaron los sujetos cuyo rol principal no fuese el ejercicio profesional de la Psicología.

En consecuencia, la muestra final estuvo formada por 77 psicólogos profesionales españoles, con una edad media de 41.44 años $(D T=9.42$, mínimo=25, máximo=64). El 31.2\% fueron hombres (68.8\% mujeres). El 27.3\% de los participantes fue licenciado/graduado, el $10.4 \%$ postgraduado, el $36.4 \%$ magister y el $26 \%$ doctor. La media de años como miembro del Colegio Oficial de Psicólogos de España fue de 13.73 
$(D T=9.30$, mínimo=0, máximo=33). Finalmente, el $87 \%$ de los participantes manifestaron utilizar artículos científicos en su toma de decisiones en la práctica profesional.

\section{Instrumentos}

La encuesta on line estuvo compuesta por dos secciones. En primer lugar, la encuesta incluyó preguntas relacionadas con la información sobre variables sociodemográficas como: sexo, edad, nivel educativo, años de experiencia como psicólogo profesional, entorno clínico (público o privado), uso de artículos científicos en la toma decisiones en la práctica profesional (Sí/no) y familiaridad con el movimiento EBP (se operacionalizó con un ítem con una escala de respuesta tipo Likert que varía desde 1=completamente en desacuerdo a 7=totalmente de acuerdo: "He oído hablar de la Practica Basada en la Evidencia”).

En segundo lugar, el instrumento incluyó un conjunto de 5 preguntas que analizaron el nivel de conocimientos sobre las cuestiones metodológicas asociadas con la PBE, tales como nivel de conocimiento de los estadísticos del tamaño del efecto, estudios meta-analíticos y listas de comprobación de la calidad metodológica de los estudios.

Conocimiento sobre cuestiones metodológicas relacionadas con la PBE: se evaluó a través de 3 bloques de preguntas: (1) Conocimiento de términos estadísticos, (2) conocimiento de listados de comprobación de la calidad de los diseños de investigación, y (3) uso de los estudios de meta-análisis en la toma de decisiones en el ejercicio profesional de la Psicología.

(1) Conocimiento de términos estadísticos, consta de 3 ítems:

a) “¿Qué términos de la siguiente lista conoce adecuadamente: desviación típica, gráfico de sedimentación, ForestPlot, ANOVA, FunnelPlot, correlación, meta-análisis, análisis de regresión, tamaño del efecto, potencia estadística?”. (Elección múltiple).

b) “Podría señalar el nombre de algún estadístico del tamaño del efecto?” (Si/no).

c) "Si su respuesta es sí, por favor, especifique el nombre de algún estadístico del tamaño del efecto" (pregunta abierta).

(2) Conocimiento de listados de control de la calidad de estudios. Consta de un único ítem con una escala de respuesta dicotómica: "Conoce algún listado de comprobación de la calidad metodológica de los estudios" ( $\mathrm{Si} / \mathrm{no})$.

(3) Uso de estudios meta-analíticos como fuente de información en la toma de decisiones profesionales. Consta de un único ítem con una escala de respuesta dicotómica: "En su práctica profesional, utiliza estudios de meta-análisis como fuente de información para tomar decisiones profesionales" (Si/no). 


\section{Procedimiento}

Se envió un correo electrónico a los Colegios Oficiales de Psicólogos de España invitándoles a participar en la encuesta on line sobre Práctica Profesional en el ámbito de la Psicología. La participación fue voluntaria y se garantizó el anonimato a los participantes. Los datos se registraron mediante un sistema CAWI (Computer Assisted Web Interviewing) en los meses de mayo-septiembre 2015. Se controló que no hubiera duplicidades en las respuestas. Se obtuvieron 113 encuestas correctamente cumplimentadas, de las cuales 77 fueron de psicólogos cuyo rol principal era el ejercicio profesional de la psicología.

\section{Análisis de datos}

Se realizaron análisis de estadísticos descriptivos (frecuencias y porcentajes). Para el cálculo de los intervalos de confianza de los porcentajes se utilizaron los métodos "score" basado en los trabajos de Newcombe (2012). Estos análisis se realizaron con el programa estadístico IBM SPSS v. 20 para Windows.

\section{RESULTADOS}

La tabla 1 muestra las respuestas de los participantes al ítem que valora su conocimiento sobre términos estadísticos. En general, se puede observar que más del $80 \%$ de los participantes afirmaron tener un conocimiento adecuado sobre los términos estadísticos de: desviación estándar (o típica), correlación e intervalos de confianza.

Tabla 1. Términos estadísticos que los participantes afirman conocer adecuadamente

\begin{tabular}{lcccc}
\hline & \multirow{2}{*}{$n$} & \multirow{2}{*}{$\%$} & \multicolumn{2}{c}{ IC 95\% } \\
\cline { 4 - 6 } & & & $\mathrm{Li}$ & $\mathrm{Ls}$ \\
\hline Desviación típica & 69 & 89.61 & 80.82 & 94.64 \\
Correlación & 65 & 84.42 & 74.71 & 90.85 \\
Intervalo de confianza & 63 & 81.82 & 71.76 & 88.85 \\
Meta-análisis & 52 & 67.53 & 56.46 & 76.94 \\
ANOVA & 46 & 59.74 & 48.58 & 69.98 \\
Análisis de regresión & 40 & 51.95 & 40.96 & 62.75 \\
Potenciaestadística & 27 & 35.06 & 25.35 & 46.20 \\
Tamaño del efecto & 24 & 31.17 & 21.93 & 42.20 \\
Gráfico de Sedimentación & 5 & 6.49 & 2.81 & 14.32 \\
Florest Plot & 1 & 1.30 & 0.23 & 0.70 \\
Funnel Plot & 1 & 1.30 & 0.23 & 0.70 \\
\hline Nota. Se puede seleccionar más de una respuesta. IC $=$ Intervalo de \\
Confianza; Li=límite inferior; Ls=límite superior
\end{tabular}

Además, la mayoría de los participantes (67.53\%) afirmaron poseer un conocimiento adecuado de los estudios de meta-análisis. No obstante, sólo el $31.17 \%$ dijo conocer adecuadamente los estadísticos del tamaño de efecto. Por lo otro lado, los gráficos que normalmente acompañan a los estudios meta-analíticos, como son el forestplot y el funnelplot, solo fueron calificados como adecuadamente conocidos por un 
porcentaje muy bajo de participantes (1.30\%). El gráfico forestplot representa el tamaño del efecto medio y su intervalo de confianza junto con los tamaños del efecto e intervalos de confianza de los estudios primarios. Por su parte, el gráfico funnetplot muestra la heterogeneidad entre los tamaños del efecto de los estudios primarios y, además, es el método de detección del sesgo de publicación de uso más frecuente en las Ciencias de la Salud. Por último, más de un tercio de los participantes (35.06\%) declaró tener un adecuado conocimiento del término potencia estadística.

En cuanto a su grado de conocimiento sobre estadísticos del tamaño del efecto, si bien el $31.17 \%$ de los participantes declaró conocer el término estadístico de tamaño del efecto, solo el $11.69 \%$ de los participantes dio el nombre de un índice del tamaño del efecto $(n=9)$. En consecuencia, hubo mayor conocimiento sobre el término estadístico "tamaño del efecto" que sobre índices del tamaño del efecto. Como se muestra en la tabla 2, los índices del tamaño del efecto más familiares para los participantes fueron los que evalúan las diferencias entre las medias de los grupos analizados (diferencia de medias estandarizada), en concreto, $d$ de Cohen, seguida de la proporción de la varianza explicada $\left(\eta^{2}\right)$ y $g$ de Glass.

Tabla 2. Índices del tamaño del efecto nombrados (respuestas de 9 participantes)

\begin{tabular}{lcccc}
\hline & \multirow{2}{*}{$n$} & \multirow{2}{*}{$\%$} & \multicolumn{2}{c}{ IC 95\% } \\
\cline { 4 - 5 } & 7 & 77.78 & 45.26 & Ls \\
\hline$d$ de Cohen & 2 & 22.22 & 6.32 & 54.74 \\
$g$ de Glass & 2 & 22.22 & 6.32 & 54.74 \\
$\eta^{2}$ & 1 & 11.11 & 1.99 & 43.50 \\
Correlación biserial & 1 & 11.11 & 1.99 & 43.50 \\
\multicolumn{2}{l}{$d$ de Cox } & Nota. 50\% de los participantes dieron el nombre de más de un \\
índice del tamaño del efecto. IC=Intervalo de Confianza; & Li=límite inferior; Ls=límite superior
\end{tabular}

Con respecto al nivel de conocimiento de listas de comprobación de la calidad metodológica de los estudios, sólo el 24.68\% (95\% CI [16.40, 35.35]) de los participantes afirmó conocer alguna lista de comprobación (Checklist). Por último, la mayoría de los participantes manifestaron haber utilizado un estudio meta-analítico en su práctica profesional, 51.95\% (IC del 95\% [40.96, 62.75]).

\section{DISCUSIÓN Y CONCLUSIONES}

La mayoría de los participantes reportaron usar estudios meta-analíticos en su práctica profesional y tener un conocimiento adecuado de los mismos. Sin embargo, reconocieron tener un pobre conocimiento del tamaño del efecto y de los gráficos que se utilizan en los estudios de meta-análisis, como el forestplot y el funnelplot, lo que podría conducir a una pobre interpretación de los resultados que, a su vez, podría afectar a su práctica profesional. Como señalan varios autores, la presentación gráfica de los 
resultados es una parte importante de un estudio de meta-análisis y se ha convertido en la principal herramienta para presentar los resultados de múltiples estudios sobre la misma pregunta de investigación (Anzures-Cabrera y Higgins 2010; Borenstein et al., 2009; Sánchez-Meca y Marín-Martínez, 2010). De esta manera, el forestplot y el funnelplot son gráficos utilizados en estudios meta-analíticos para presentar estimaciones de tamaño de efecto medio (forestplot) y evaluar el sesgo de publicación (funnelplot), respectivamente.

Como se ha comentado, el sesgo de publicación es una amenaza importante para la validez de los estudios meta-analíticos, ya que las estimaciones derivadas metaanalíticamente podrían ser inexactas, normalmente sobreestimadas. De hecho, como señalan Kepes, Banks, y Oh (2014), dada la influencia de los estudios meta-analíticos sobre las direcciones de investigación futuras y la PBE, el sesgo de publicación se ha referido como "el talón de Aquiles de las revisiones sistemáticas" (Torgerson, 2006), "la kryptonita de la práctica basada en la evidencia" (Banks y McDaniel 2011), y el "antagonista de la formulación efectiva de políticas" (Banks, Kepes, y Banks, 2012). Como se ha comentado, en Ciencias de la Salud, el funnelplot se utiliza como método de detección del sesgo de publicación (Sterneet et al., 2005) junto con el método de trim and fill en el que se imputan tamaños del efecto de magnitud baja o nula, que son los que no logran ser publicados.

Por lo tanto, los investigadores, los académicos y los profesionales deberían tener un conocimiento adecuado de este gráfico, el cual permite al lector evaluar si los resultados del estudio de meta-análisis podrían ser reflejo de un tamaño del efecto sobreestimado (Bauer, 2007). En consecuencia, dado el escaso conocimiento detectado en el presente trabajo, se requieren programas de formación en metodología que favorezcan la adquisición de conocimientos y habilidades necesarias en la valoración crítica de los resultados de estudios de meta-análisis para que los psicólogos profesionales puedan valorar la calidad y relevancia de la evidencia empírica aportados por los estudios meta-analíticos (Spring, 2007; Walker y London, 2007).

En cuanto al tipo de índices del tamaño de efecto que conocen los participantes, los índices más frecuentemente mencionados fueron los índices de la familia de la diferencia de medias estandarizada y $\eta^{2}$ (índices paramétricos del tamaño del efecto). Como se ha comentado, justamente los índices de la familia de la diferencia de medias estandarizada y $\eta^{2}$ han sido criticados por su falta de robustez frente a los valores atípicos o la violación del supuesto de normalidad de la variable, y por su inestabilidad a las violaciones de los supuestos estadísticos; los cuales son comunes en la práctica (Algina, Keselman y Penfield, 2005; Erceg-Hurn y Mirosevich, 2008; Grissom y Kim, 2001; Kline, 2013; Peng y Chen, 2014; Wang, y Thompson, 2007).

Finalmente, en cuanto a las listas de comprobación de la calidad metodológica de los estudios, una vez más la mayoría de los participantes dijeron no tener 
conocimiento sobre las mismas. Sin embargo, existen listas de verificación para estudios primarios (e.g., CONSORT) y para estudios meta-analíticos (e.g., AMSTAR o PRISMA-NMA). Estas listas de verificación son útiles para los psicólogos profesionales si desean valorar la calidad de la evidencia de la investigación sobre la efectividad de los tratamientos (Spring, 2007).

En la línea de previos estudios el presente trabajo aporta evidencia sobre la necesidad de formación estadística de los psicólogos profesionales, dado el pobre conocimiento sobre los tamaños del efecto, estudios de meta-análisis y las listas de comprobación de la calidad metodológica de los estudios. La PBE requiere tener un conocimiento adecuado de los fundamentos de la metodología de investigación para poder evaluar críticamente las evidenticas científicas que los estudios reportan en sus informes (Bauer, 2007). El pobre conocimiento metodológico podría influir en las conclusiones que los profesionales extraen de los estudios científicos, lo que podría poner en riesgo la calidad de las intervenciones psicológicas.

Finalmente, deben mencionarse algunas limitaciones de este estudio. La baja tasa de respuesta podría afectar la representatividad de la muestra y, por lo tanto, la generalización de los hallazgos entre los psicólogos profesionales españoles. Sin embargo, es posible que los participantes que respondieron a la encuesta se sintieran más seguros sobre sus conocimientos estadísticos y metodológicos que los que no respondieron. En este caso, los resultados podrían sobreestimar el nivel de conocimientos de los términos estadísticos analizados. Además, es posible que haya habido un efecto de deseabilidad social, como es habitual cuando los datos se recogen utilizando instrumentos de auto-informe. Por ejemplo, el porcentaje de participantes que dijeron tener un conocimiento adecuado del término "tamaño del efecto" fue mayor que el porcentaje de participantes que afirmaron poder nombrar un estadístico del tamaño del efecto. Y, a su vez, el porcentaje de los participantes que dijeron que podían nombrar algún índice del tamaño del efecto fue mayor que el porcentaje de los participantes que realmente dio el nombre. Sin embargo, nuestros resultados concuerdan con los hallazgos de estudios previos sobre el nivel de conocimiento del tamaño del efecto y los estudios meta-analíticos en psicólogos académicos españoles (Badenes-Ribera et al., 2016) y en psicólogos académicos italianos y chilenos (Badenes-Ribera, 2016).

Todo esto nos lleva a indicar la necesidad de formación metodológica de los profesionales de la Psicología para mejorar la práctica profesional. Como se decía, la PBE requiere de los profesionales que evalúen críticamente los resultados de la investigación psicológica. Para ello, se requiere una formación en conceptos estadísticos, metodología de diseño de investigación, interpretación de los resultados de las pruebas de inferencia estadística y de los estudios de meta-análisis (Bauer, 2007; Frías-Navarro y Pascual-Llobell, 2003; Spring, 2007). 


\section{Agradecimientos}

Este artículo se basa en la Tesis Doctoral con Mención Internacional defendida por Laura Badenes-Ribera en la Facultad de Psicología de la Universidad de Valencia el 15 de diciembre de 2016, subvencionda por el "Programa VALi+d per a Personal Investigador en Formació de caràcter Pre-doctoral” (ACIF/2013/167) de la Conselleria d’Educació, Cultura i Esport, Generalitat Valenciana (España).

\section{REFERENCIAS}

Algina, J., Keselman, H.J., y Penfield, R.D. (2005). An alternative to Cohen's standardized mean difference effect size: a robust parameter and confidence interval in the two independent groups case. Psychological Methods, 10, 17-328. doi:10.1037/1082-989X.10.3.317

American Psychological Association (2005). Policy Statement on Evidence-Based Practice in Psychology. Washington, DC: Author.

American Psychological Association (2006). Evidence-based practice in psychology: APA Presidential Task Force on evidence-based practice. American Psychologist, 61, 271285. doi:10.1037/0003-066X.61.4.271

American Psychological Association (2010). Publication Manual of the American Psychological Association (6th ed.). Washington, DC: Author.

Anzures-Cabrera, J., y Higgins, J.P.T. (2010). Graphical displays for meta-analysis: An overview with suggestions for practice. Research Synthesis Methods, 1, 66-80. doi:10.1002/jrsm.6

Babione, J.M. (2010). Evidence-Based Practice in Psychology: An ethical framework for graduate education, clinical training, and maintaining professional competence. Ethics \& Behavior, 20, 443-453. doi:10.1080/10508422.2010.521446

Badenes-Ribera, L. (2016). Tamaño del efecto y su intervalo de confianza y meta-análisis en Psicología. Tesis Doctoral con Mención Internacional. Univesitat de València.

Badenes-Ribera, L., Frías-Navarro, D., Monterde-i-Bort, H., y Pascual-Soler, M. (2013, Febrero). Informar e interpretar el tamaño del efecto en Psicología y Educación. Comunicación escrita presentada al XIV Congreso Virtual de Psiquiatria.com. Interpsiquis, Palma de Mallorca, Spain.

Badenes-Ribera, L., Frías-Navarro, D., Pascual-Soler, M., y Monterde-i-Bort, H. (2016). Knowledge level on effect size statistics, confidence intervals and meta-analysis in Spanish academic psychologists. Psicothema, 28, 448-456. doi:10.7334/psicothema2016.24

Banks, G.C., Kepes, S., y Banks, K.P. (2012). Publication bias: The antagonist of meta-analytic reviews and effective policymaking. Educational Evaluation and Policy Analysis, 34, 259-277. doi:10.3102/0162373712446144

Banks, G.C., y McDaniel, M.A. (2011). The kryptonite of evidence based I-O psychology. Industrial and Organizational Psychology: Perspectives on Science and Practice, 4, 4044. doi:10.1111/j.1754-9434.2010.01292.x.

Bauer, R.M. (2007). Evidence-based practice in Psychology: implications for research and research training. Journal of Clinical Psychology, 63, 685-694. doi:10.1002/jclp.20374

Beyth-Maron, R., Fidler, F., y Cumming, G. (2008). Statistical cognition: Towards evidence-based practice in statistics and statistics education. Statistics Education Research Journal, 7, 20-39.

Borenstein, M., Hedges, L.V., Higgins, J.P.T., y Rothstein, H. (2009). Introduction to Metaanalysis. Chichester: Wiley. 
Botella, J., y Sánchez-Meca, J. (2015). Meta-análisis en Ciencias Sociales y de la Salud. Madrid, Spain: Síntesis.

Clarke, M., y Clarke, T. (2000). A study of the references used in Cochrane protocols and reviews. Three bibles, three dictionaries, and nearly 25,000 other things. International Journal of Technology Assessment in Heath Care, 16, 907-909.

Cumming, G. (2012). Understanding the new statistics: Effect sizes, confidence intervals, and meta-analysis. New York, NY: Routledge.

Cumming, G., Fidler, F., Kalinowski, P., y Lai, J. (2012). The statistical recommendations of the American Psychological Association publication manual: Effect sizes, confidence intervals, and meta-analysis. Australian Journal of Psychology, 64, 138-146. doi:10.1111/j.1742-9536.2011.00037.x

Daset, L.R., y Cracco, C. (2013). Psicología Basada en la Evidencia: algunas cuestiones básicas y una aproximación a través de una revisión bibliográfica. Ciencias Psicológicas, 7, 209220.

Erceg-Hurn, D.M., y Mirosevich, V.M. (2008). Modern robust statistical methods: An easy way to maximize the accuracy and power of your research. American Psychologist, 63, 591601. doi:10.1037/0003-066X.63.7.591

Faulkner, C., Fidler, F., y Cumming, G. (2008). The value of RCT evidence depends on the quality of statistical analysis. Behavior Research and Therapy, 46, 270-281. doi:10.1016/j.brat.2007.12.001

Ferguson, C.J. (2009). An effect size primer: a guide for clinicians and researchers. Professional Psychology: Research and Practice, 40, 532-538. doi:10.1037/a00158084

Ferguson, C.J., y Brannick, M.T. (2011). Publication bias in psychological science: Prevalence, methods for identifying and controlling, and implications for the use of meta-analyses. Psychological Methods, 17, 120-128. doi:10.1037/a0024445

Frías-Navarro, D., y Pascual-Llobell, J. (2003). Psicología clínica basada en pruebas: Efecto del tratamiento. Papeles del Psicólogo, 85, 11-118.

Grissom, R.J., y Kim, J.J. (2001). Review of assumptions and problems in the appropriate conceptualization of effect size. Psychological Methods, 6, 135-146. doi:10.1037/1082-989X.6.2.135

Grissom, R.J., y Kim, J.J. (2012). Effect sizes for research. New York, USA: Routledge.

Ioannidis, J.P.A. (2011). Meta-research: The art of getting it wrong. Research Synthesis Methods, 1, 169-184. doi:10.1002/jrsm.19

Kepes, S., Banks, G.C., y Oh, I.S. (2014). Avoiding bias in publication bias research: The value of "null" findings. Journal of Business and Psychology, 29, 183-203. doi: 10.1007/s10869-012-9279-0

Kicinski, M. (2013). Publication Bias in Recent Meta-Analyses. PLoSOne, 8(11), e81823.

Kicinski, M., Springate, D.A., y Kontopantelis, E. (2015). Publication bias in meta-analyses from the Cochrane Data base of Systematic Reviews. Statistics in Medicine, 34, 2781-2795. doi:10.1002/sim.6525

Kline, R.B. (2013). Beyond significance testing: Statistic reform in the behavioral sciences. Washington, DC: American Psychological Association: Washington, DC.

Newcombe, R.G. (2012). Confidence Intervals for Proportions and Related Mesuares of Efect Size. Boca Raton, FL: CRC Press.

Peng, C.Y.J., y Chen, L.T. (2014). Beyond Cohen's d: alternative effect size measures for between subject designs. The Journal of Experimental Education, 82, 22-50. doi:10.1080/00220973.2012.745471 
Rothstein, H.R., Sutton, A.J., y Borenstein, M. (Eds.) (2005). Publication bias in meta-analysis: Prevention, assessment, and adjustments. New York, NY: Wiley. doi:10.1002/0470870168

Sánchez-Meca, J., Boruch, R.F., Petrosino, A., y Rosa-Alcázar, A.I. (2002). La Colaboración Campbell y la Práctica basada en la Evidencia. Papeles del Psicólogo, 83, 44-48.

Sánchez-Meca, J., y Marín-Martínez, F. (2010). Meta-analysis in psychological research. International Journal of Psychological Research, 3, 150-162.

Spring, B. (2007). Evidence-based practice in clinical psychology: What it is, why it matters; what you need to know. Journal of Clinical Psychology, 63, 611-631. doi:10.1002/jclip.20373

Sterne, J.A., Gavaghan, D., y Egger, M. (2005). The funnel plot. En H.R. Rothstein, A.J. Sutton, y M. Borenstein (Eds.), Publication bias in metaanalysis: Prevention, assessment and adjustments (pp. 75-98). Chichester, UK: Wiley.

Torgerson, C.J. (2006). Publication bias: The Achilles' heel of systematic reviews? British Journal of Educational Studies, 54, 89-102. doi:110.1111/j.1467-8527.2006.00332.x.

Walker, B.B., y London, S. (2007). Novel tools and resources for evidence-based practice in Psychology. Journal of Clinical Psychology, 63, 633-642. doi:10.1002/jclp.20377

Wang, Z., y Thompson, B. (2007). Is the Pearson $\mathrm{r}^{2}$ biased, and if so, what is the best correction formula? Journal of Experimental Education, 75, 109-125. doi:10.3200/JEXE.75.2.109125

Recibido: 10 de marzo de 2017 Recepción Modificaciones: 14 de junio de 2017 Aceptado: 20 de junio de 2017 